\title{
Inventaire de la flore fongique et identification des différents horizons du profil au sein de la bananeraie de TADMAIT
}

\author{
Juliette K.Y. DEDI ${ }^{*}$, Kouassi René ALLOU $^{2}$ et Atcho OTCHOUMOU ${ }^{2}$ \\ ${ }^{1}$ Laboratoire de Biologie et Amélioration des Productions Végétales, Université Nangui Abrogoua, \\ UFR-SN 01 BP 8133 Abidjan 01, Côte d'Ivoire. \\ ${ }^{2}$ Chercheur en Phytopathologie et Entomologie au CNRA Vacataire à l'Université Nangui Abrogoua, \\ Station Marc Delorme, 07 BP 13 Abidjan 07, Côte d'Ivoire. \\ ${ }^{2}$ Laboratoire de Biologie et Cytologie Animale, Université Nangui Abrogoua, \\ UFR-SN, 02 BP 801 Abidjan 02, Côte d'Ivoire. \\ *Auteur correspondant ; E-mail : mmededijuliette@yahoo.fr
}

\section{RESUMÉ}

L'objectif de ce travail a été de faire une étude mycologique au sein de la bananeraie parce qu'elle n'a pas été faite avant l'installation de celle-ci et confirmer par une étude physico-chimique des horizons que le site est bien propice à la culture du bananier. Des prélèvements ont été effectués d'une part à différents endroits au sein de la bananeraie à l'aide d'une truelle, mis séparément dans des sachets neufs étiquetés et conservés au frais. Différentes suspensions dilutions ont été préparées à partir des échantillons prélevés et ces dernières ont permi l'inoculation du milieu de culture Potato Dextrose Agar (PDA). Des champignons se sont développés sur le milieu. Ils ont été isolés, purifiés puis identifiés au microscope. Ainsi nous avons pu caractériser la flore fongique de la bananeraie. Vingt champignons ont été révélés. Ce sont: Achlya sp., Acremonium sp., Aspergillus sp., Basipetospora sp., Botrytis sp., Brachysporium sp., Candida sp., Chrysosporium sp., Fusarium oxysporum, Gliocladium sp., Monilia sp., Olpitrichum sp., Paecilomyces sp., Penicillium sp., Pythium de Baryanum, Rhizopus sp., Staphylotrichum sp., Streptomyces sp., Trichocladium sp. et Trichoderma sp. La présence de Fusarium oxysporum peut être catastrophique pour la bananeraie si les conditions climatiques sont réunies pour son développement car, il est responsable de la maladie de Panama qui entraîne la mort des bananiers après flétrissement. D'autre part, un profil de sol ouvert dans la bananeraie a permis de prélever 5 échantillons de sol, de bas en haut pour éviter les contaminations des échantillons. Ces échantillons ont été mis à sécher à l'étuve pendant 5 jours à $37^{\circ} \mathrm{C}$ au laboratoire bien broyés puis passés au tamis métallique dans la zone du bec Benzène. L'analyse physico-chimique du sol prélevé a montré qu'il présente des propriétés favorables à la culture du bananier : un pH basique (entre 7,5 et 8,5), une très faible salinité $(129,90 \mu \mathrm{mhos} / \mathrm{cm}$ à l'horizon C et $211,36 \mu \mathrm{mhos} / \mathrm{cm}$ à l'horizon R) une structure légère du sol (alternance de texture tout au long du profil) et un bon drainage. Cette étude a permis la caractérisation de la flore fongique de la bananeraie tout en déterminant la nature du sol.

(C) 2015 International Formulae Group. All rights reserved.

Mots clés : Champignons, dosage, PDA, propriétés physico-chimiques, horizon.

\section{INTRODUCTION}

La banane dessert figure parmi les fruits tropicaux les plus importants et est le fruit le plus produit au monde : 106,5 millions de tonnes en 2011 (PACIR, 2013). Elle constitue la deuxième production mondiale de fruits : 50 millions de tonnes environ par an (Cirad, 2010). Les exportations de bananes se 
situent autour de 240000 tonnes/an pour une superficie cultivée de près de 6000 hectares.

Sa production exige un sol léger, bien drainé, profond, légèrement acide, bien aéré dont les $20 \mathrm{~cm}$ supérieurs riches en minéraux et en matière organique. Les sols profonds, limoneux et bien drainés conviennent le mieux pour la culture du bananier. Les éléments N.P.K., $\mathrm{Ca}$ et $\mathrm{Mg}$ sont indispensables pour atteindre un bon niveau de développement et une production élevée. Le $\mathrm{pH}$ peut varier de 4,0 à 8,0 (Swennen et Vuylsteke, 2001).

En climat chaud et humide, une pluviométrie mensuelle de 125 à $150 \mathrm{~mm}$ couvre généralement les besoins en eau (Cirad, 2002). Le bananier, quel qu'il soit, peut être cultivé de manière intensive. La bananeraie est considérée comme une culture pérenne alors que le bananier, en tant qu'individu isolé de sa souche, n'est pas une plante pérenne car il disparaît après avoir produit ses fruits. Cependant, la souche dont il provient, produit des rejets faisant de chaque "touffe" de bananier un élément récurrent (FAO, 2003). C'est à partir d'un rejet latéral sain qu'un nouveau cycle de culture redémarre grâce à la technique de bouturage après la destruction de la plantation préexistante. Cette méthode de reproduction par multiplication végétative a deux inconvénients majeurs : une faible diversité des variétés cultivées, d'où des risques accrus de parasitose et la propagation par les rejets de parasites (notamment les nématodes). Les plantations industrielles ont donc tendance à utiliser une autre technique : le vitro plant. Les plants de bananier issus de cette technique limitent considérablement la dissémination des ravageurs et des maladies par l'intermédiaire du matériel de plantation (Cabrera et al., 2010).

La culture du bananier existait déjà en Algérie depuis le début du $19^{\mathrm{e}}$ siècle au nord à Ghazaouet et au sud à Biskra (Ganry, 1987). Guidés par l'intérêt qu'ils portaient aux vignes et aux agrumes, les colons ont renoncé à ces essais. Cette culture fut réintroduite en Algérie en 1988 et elle se fait sous serre, ce qui nécessite un investissement considérable. Mais la serre peut être vite amortie vu le taux de rentabilité de la culture. Les variétés cultivées en Algérie appartiennent à l'espèce Musa accuminata, essentiellement au groupe Cavendish qui renferme les variétés grande naine, petite naine, williams et le poyo. Le bananier est menacé. Outre les rafales de vent, les tempêtes, les cyclones, le bananier a de redoutables ennemis. De lourdes menaces parasitaires pèsent sur la production de cette culture. Parmi ces menaces, les maladies fongiques, les maladies bactériennes, les nématodes, les maladies virales et les insectes sont signalés dans les zones de culture de la banane (Baudouin et al., 2002). Les maladies virales sont toujours des infections généralisées de la plante, excepté les méristèmes. Elles sont transmissibles par le matériel végétal et en particulier au cours de la multiplication in vitro, si des précautions draconiennes ne sont pas mises en œuvre. Cependant, la transmission peut se faire aisément à partir d'hôtes réservoir et d'agents vecteurs (Lassoudière, 2007). La cercosporiose noire (Maladie des Raies Noires, MRN, causée par Mycosphaerella fijiensis) et la cercosporiose jaune (Maladie de Sigatoka, MS, causée par Mycosphaerella musicola) constituent deux contraintes majeures pour la production de bananes dessert destinées à l'exportation. Ces maladies provoquent des nécroses foliaires, des pertes de rendement et surtout la maturation précoce des fruits qui les rend impropres à l'exportation (Lapeyre et al., 2010). Les prévisions de la filière pour 2014 étaient d'étendre le verger de 8900 ha, avec des rendements de 50 tonnes/ha et une production exportée de 420000 tonnes. Pour atteindre ces objectifs, il fallait lever certaines contraintes d'ordre phytosanitaire, notamment celles liées aux nématodes, à la cercosporiose noire ou aux charançons (CNRA, 2015). 
L'un des problèmes actuels provient de la très grande importance de la variété Cavendish, au moins pour ce qui est des bananes qui font l'objet de commerce international. En effet, la diversité génétique étant très faible, le développement d'un parasite ou autre maladie touchant cette variété pourrait avoir des conséquences catastrophiques sur la production mondiale (Gilles, 2012). De fait, si la variété Cavendish a pu prendre la place de la variété Gros Michel, c'est parce qu'elle était résistante à la maladie de Panama. Mais le manque de variétés rend toute monoculture très susceptible aux maladies (Gilles, 2012). Or une souche dite R4 de Fusarium à laquelle la variété Cavendish est sensible est apparue en Asie, tandis qu'un autre champignon vecteur de la maladie des raies noires, qui touche également la variété Cavendish, se développe sur le continent américain. Ces champignons suscitent l'inquiétude et, outre la recherche de solutions phytosanitaires, pourraient pousser à développer la culture d'autres variétés. Les bananiers même sous serre, sont également exposés aux mêmes ennemis. Les fortes densités favorisent, par les microclimats qu'elles créent, le développement des champignons parasites, des araignées et des insectes. La bananeraie de Tadmait est-elle sous la menace d'éventuels parasites en particulier des champignons ? Sachant que la plantation existe depuis plus de deux années et qu'aucune étude mycologique préalable n'a été faite. Le but de notre travail est de déterminer la flore fongique de la bananeraie sous- serre et de faire l'analyse physico-chimique du sol qui a un effet direct sur le développement de la végétation, sur la nature et la biomasse des champignons.

\section{MATERIEL ET METHODES Matériel}

L'étude a été menée en 1990 -1991 dans la bananeraie sous serre de Tadmait. Le sol prélevé entre et en dehors des différents plants de bananes puis au niveau des horizons du profil creusé au sein de la bananeraie constituent nôtre matériel d'étude.

\section{Etude pédologique \\ Prélèvements au niveau des horizons du profil}

Un kilogramme de terre a été prélevé au niveau de chaque horizon en commençant par ceux de profondeur tout en évitant les lieux de chevauchements. Cinq échantillons d'un kilogramme ont été ramenés au laboratoire. La profondeur du profil était de $200 \mathrm{~cm}$.

Tous les prélèvements ont été faits dans des conditions rigoureuses d'asepsie. Cependant, il faut éviter tout contact de la terre avec les doigts et utiliser du matériel flambé ou passé à l'alcool à $90^{\circ}$.

\section{Préparation des échantillons prélevés}

$\mathrm{Au}$ laboratoire, avant l'analyse, les échantillons prélevés au niveau des horizons ont été mis à sécher à l'étuve pendant 5 jours à environ $37^{\circ} \mathrm{C}$ jusqu'à ce qu'ils soient secs. Dans la zone stérile du bec Bunsen, ils ont été broyés puis passés au tamis métallique de 2 $\mathrm{mm}$, préalablement aseptisé par passage rapide dans la flamme du bec Bunsen. Les produits du tamisage ont été conservés dans des sachets fermés et disposés sur une étagère à la température du laboratoire.

\section{Technique d'analyse}

-La détermination du $\mathbf{p H}$ du sol a été faite selon la méthode électro métrique (Black et Evan, 1965). La mesure du $\mathrm{pH}_{\text {eau }}$ a été faite par la lecture directe sur $\mathrm{pH}$-mètre, dans une suspension sol /eau de 1/2,5. Quant au pH la lecture s'est également faite directement sur pH- mètre après l'ajout de $3,72 \mathrm{~g}$ de KCL pur à la suspension aqueuse sol/eau précédente après 2 à 3 minutes d'agitation afin de dissoudre le sel.

- La conductivité électrique a été réalisée grâce à la méthode Bonfils (1967) qui consiste à introduire l'électrode du conductimètre au centre du récipient contenant l'extrait de sol 
au 1/5 auquel a été ajouté de l'hexamétaphosphate de sodium à $0,1 \%$ afin d'éviter la précipitation du calcaire. La conductivité électrique d'une solution dépend de sa concentration en ion et de sa température.

- L'humidité hygroscopique est la différence de poids d'un échantillon avant et après séchage à $105{ }^{\circ} \mathrm{C}$ pendant 24 heures et est déterminée par la formule suivante :

$\mathrm{H}=\frac{\mathrm{a}}{\mathrm{b}} * 100 \quad \mathrm{a}=$ masse du sol avant séchage (en gramme) ; b = masse du sol sec ( $105^{\circ} \mathrm{C}$ à l'étuve)

- Le carbone organique a été dosé à partir de la méthode de Duchaufour (1970) qui se fonde sur l'oxydation du carbone organique par du bichromate de potassium en présence d'acide sulfurique à chaud. Par la suite, l'excès de bichromate de potassium est titré par une solution de sel de Mohr à 0,2 $\mathrm{N}$ en présence de fluore de sodium et de diphénylamine. Le taux de matière organique est égal : $\quad$ M.O

$(\%)=\mathrm{CO}(\%) \times 1,72$

$\mathrm{M} . \mathrm{O}=$ Matière Organique

$\mathrm{CO}=$

Carbone organique

\section{- Dosage des calcaires total et actif}

Le principe de dosage du calcaire total par la méthode volumétrique (Baize, 2000) est basé sur la mesure du $\mathrm{CO}_{2}$ dégagé du calcaire (CaCO3) se trouvant dans $0,5 \mathrm{~g}$ de terre fine neutralisée par $5 \mathrm{ml}$ d'acide chlorhydrique $(\mathrm{HCl})$. La réaction est la suivante :

$\mathrm{CACO}_{3}+2 \mathrm{HCL} \rightarrow \mathrm{CACL}_{2}+$ $\mathrm{CO}_{2}+\mathrm{H}_{2} \mathrm{O}$

L'appareil de mesure est appelé calcimètre de Bernard.

Le calcaire actif représente la partie du calcaire facilement solubilisable. Son dosage s'est effectué selon la méthode de Drouineau (1940) suivant la réaction :

$\mathrm{CACO}_{3}+(\mathrm{NH} 4 \mathrm{COO})_{2} \rightarrow \mathrm{CA}(\mathrm{COO})_{2}$ $+(\mathrm{NH} 4)_{2} \mathrm{CO}_{3}$

L'excès de (NH4COO $)_{2}$ est titré par du permanganate de potassium à $0,2 \mathrm{~N}$.
- Le dosage des anions repose sur la neutralisation d'un volume de solution par de l'acide minéral en présence de la phénolphtaléine pour les carbonates et du méthyl orange pour les bicarbonates. Par argentimétrie, les chlorures sont précipités par du nitrate d'argent à $0,02 \mathrm{~N}$ en présence du chromate de potassium à $5 \%$.

- Le dosage de l'azote a été fait selon la méthode Kjeldah (1883) qui se fonde sur :

- la minéralisation de l'azote par de l'acide sulfurique concentré en présence de catalyseur au sélénium pour activer la réaction ;

. l'azote passé à l'état d'ammoniaque est fixé par l'acide sulfurique en excès sous forme de sulfate d'ammonium (SO4(NH4)2);

. l'azote qui est lié à l'acide sulfurique peut par la suite être déplacé et dosé par une solution d'acide sulfurique à $0,014 \mathrm{~N}$.

- L'analyse granulométrique à été réalisée selon la méthode internationale. Elle s'effectue sur une prise d'essai de terre fine (éléments $<2 \mathrm{~mm}$ ). Cette méthode consiste en un prétraitement de l'échantillon par une solution d'hexamétaphosphate de sodium et par une agitation énergique et prolongée afin de disperser les particules de sol. Les éléments fins (argile, limon fin et grossier) ont été prélevés à l'aide de la pipette de Robinson. Les sables fins et grossiers sont déterminés par lavage. La texture du sol est déterminée grâce au triangle des textures de la «soil taxonomy » (Duchaufour, 1970).

\section{Etude mycologique \\ Prélèvements dans la bananeraie}

Sous les pieds de bananiers, à l'aide d'une truelle, la couche superficielle du sol est éliminée afin d'éviter les champignons envahisseurs. Après homogénéisation, $500 \mathrm{~g}$ de sol sont prélevés à raison de trois échantillons à $5 \mathrm{~cm}$ de profondeur à l'extérieur de la serre, à l'intérieur de la serre, à l'entrée et au fond mais en dehors des plants de bananiers, à $30 \mathrm{~cm}$ du pied de chaque 
variété de bananes, entre deux plants de la même variété et au centre d'un carré de quatre plants de la même variété et sont mis séparément dans des sachets neufs affectés d'une étiquette indiquant la date, le lieu et le numéro de l'échantillon. Tous les échantillons sont conservés au frais dans un réfrigérateur.

\section{Isolement des souches de champignons telluriques}

Les prélèvements des échantillons de sol pour la recherche de champignons microscopiques ont été effectués à Tadmait au sein de la bananeraie sous serre. Une série de dilutions du sol au 1/10 a été effectuée selon la méthode Pochon et Tardieux (1962). Seulement deux dilutions $10^{-4}$ et $10^{-5}$ ont été retenues pour l'ensemencement du milieu de culture Potato Dextrose Agar (PDA) choisi pour l'isolement sélectif des champignons. L'acide citrique (bactéricide : 0,25 g / $250 \mathrm{ml}$ d'extrait) est ajouté au milieu au moment de sa distribution dans les boîtes de Pétri sous la hotte en présence de la flamme du bec Bunsen.

Toutes les opérations sont faites dans des conditions d'asepsie rigoureuses en récipients stérilisés. L'ensemencement a été effectué à la surface du milieu PDA par étalement de $1 \mathrm{ml}$ de l'inoculum. L'incubation a duré sept jours. Trois essais à raison de trois répétitions par essai ont été faits.

\section{Isolats fongiques}

Après incubation, une mycoflore variée s'est développée. Afin de purifier les souches et les maintenir en culture pure, deux à trois repiquages successifs sur milieu PDA ont été effectués. Une fois purifiées, les souches sont identifiées sur la base de leurs caractères culturaux et morphologiques (Barnett et Hunter, 1972 ; Champion, 1997). Ces données ont permis la détermination du genre de chaque souche.

\section{Pourcentage d'apparition de chacun des champignons}

Le calcul du pourcentage s'est fait selon la formule suivante :

$$
\begin{aligned}
& \% \text { apparition }=\frac{\text { NAC }}{-------~} \text { x } 100 \\
& \%=\text { pourcentage } ; \quad \text { NAC }=\text { Nombre }
\end{aligned}
$$

\section{Analyse statistique}

Une analyse de variance des résultats obtenus a été réalisée à l'aide du logiciel SAS (Statistical Analysis System). Le classement des moyennes a été effectué au seuil de 5\% selon le test de Newman-Keuls.

\section{RESULTATS}

\section{Les champignons identifiés}

Les champignons isolés qui ont pu être identifiés sont au nombre de vingt. Ce sont Achlya sp., Acremonium sp., Aspergillus sp., Basipetospora sp., Botrytis sp., Brachysporium sp., Candida sp., Chrysosporium sp., Fusarium oxysporum, Gliocladium sp., Monilia sp., Olpitrichum sp., Paecilomyces sp., Penicillium sp., Pythium de Baryanum, Rhizopus sp., Staphylotrichum sp., Streptomyces sp., Trichocladium sp. et Trichoderma sp. Ils étaient présents sur l'ensemble des échantillons. Cependant, Achlya sp., Aspergillus sp., Candida sp., Botrytis sp., Monilia sp., Pythium de Baryanum, Fusarium oxysporum et Rhizopus $s p$. ont été isolés plus à proximité des plants de bananiers. Le pourcentage d'apparition des champignons est réparti dans le Tableau 1.

\section{Analyse des différents horizons}

L'humidité passe de $6,84 \%$ à $15,04 \%$ de horizon $\mathrm{A}$ à $\mathrm{R}$ avec un maximum de $16,32 \%$ à l'horizon $\mathrm{S}$. Les cinq horizons sont caractérisés chacun par une absence de carbonate, une conductibilité de $0,0001 \mu \mathrm{mhos} / \mathrm{cm}$, un taux de bicarbonate et de chlorure de $0,09 \%$ et de $0,01 \%$. Le taux de calcaire total se situe entre $63,25 \%$ et $68,57 \%$ respectivement à l'horizon $\mathrm{A}$ et $\mathrm{S}$ (Tableau 2). 
Le taux de carbone est de $0,26 \%$ à l'horizon $\mathrm{A}$ et de $1,83 \%$ à l'horizon R. Le pourcentage de matière organique passe de 0,45 à $3,1 \%$ respectivement de horizon $\mathrm{A}$ à $\mathrm{R}$. La quantité d'azote en $\mathrm{E}$ est de $0,21 \%$ alors qu'en surface en B elle est de $0,4 \%$. Le rapport $\mathrm{C} / \mathrm{N}$ est de 0,76 à l'horizon $\mathrm{A}$ et de 8,74 à l'horizon $\mathrm{R}$. Le diagramme des textures nous donne une alternance de texture limoneuse et limono- sableuse (Tableau 3).

\section{Description morphologique du profil selon la méthode de Magnien (1980) \\ Environnement du profil}

Le profil est localisé au fond de la bananeraie de Tadmait (Wilaya de Tizi Ouzou) avec une latitude : $36^{\circ} 45^{\prime} 2^{\prime \prime}$ Est et une longitude : $3^{\circ} 53^{\prime} 50^{\prime \prime}$ Nord. L'altitude est de $50 \mathrm{~cm}$ avec une pente nulle. La végétation est culturale (bananeraie). La roche mère est $\mathrm{du}$ sable. Les critères de subdivisions des horizons étaient la netteté et la régularité.

Description des horizons du profil A : $0-42,5 \mathrm{~cm}$

Structure légère et particulaire, présence d'éléments grossiers, très forte effervescence à l'HCl. Action à l'eau oxygénée: positive, très poreux, absence de revêtement compact, présence abondante de fines racines, pas de traces d'activités biologiques, marron-clair.
B : $42,5 \mathrm{~cm}-77,5 \mathrm{~cm}$

Structure légère et particulaire, très forte effervescence à l'HCl. Action à l'eau oxygénée : positive, très poreux, peu compact, présence de racines fines dont quelques unes sont en décomposition, marron -clair.

S: $77,5 \mathrm{~cm}-93,5 \mathrm{~cm}$

Structure légère et particulaire, présence de coquilles d'escargots, très forte effervescence à l'HCl, action à l'eau oxygénée: positive, très poreux, meuble, présence abondante de fines racines, marron clair.

C : $93,5 \mathrm{~cm}-114,5 \mathrm{~cm}$

Structure fine et particulaire, très forte effervescence à l'HCL, présence : de cendre, de tâches rouges orangées, d'éléments grossiers. Action à l'eau oxygénée : positive, meuble, présence de fines racines, les racines mortes sont en décomposition, marron -clair.

$\mathrm{R}: 114,5 \mathrm{~cm}$ et plus

Structure grossière, très forte effervescence à l'HCL. Action à l'eau oxygénée: positive, présence de matière organique (cendre), de micas abondants, très poreux, meuble, absence de revêtements, racines rares, très riche en éléments grossiers, pas de traces d'activités biologiques, marron clair.

Tableau 1: Pourcentage d'apparition de chacun des champignons sur l'ensemble des échantillons.

\begin{tabular}{lc}
\hline Champignons & \% d'apparition des champignons sur l'ensemble des échantillons \\
\hline Achlya sp. & $20 \%$ \\
Acremonium sp. & $15 \%$ \\
Aspergillus sp. & $17 \%$ \\
Basipetospora sp. & $5 \%$ \\
Botrytis sp. & $10 \%$ \\
Brachysporium sp. & $5 \%$ \\
Candida sp. & $10 \%$ \\
Chrysosporium sp. & $10 \%$ \\
\hline
\end{tabular}




\begin{tabular}{lc}
\hline Fusarium oxysporum & $25 \%$ \\
Gliocladium sp. & $10 \%$ \\
Monilia sp. & $30 \%$ \\
Olpitrichum sp. & $20 \%$ \\
Paecilomyces sp. & $15 \%$ \\
Penicillium sp. & $13 \%$ \\
Pythium de Baryanum & $15 \%$ \\
Rhizopus sp. & $11 \%$ \\
Staphylotrichum sp. & $6 \%$ \\
Streptomyces $\mathrm{sp}$. & $7 \%$ \\
Trichocladium $\mathrm{sp}$. & $12 \%$ \\
Trichoderma $\mathrm{sp}$. & $22 \%$ \\
\hline
\end{tabular}

\section{DISCUSSION}

Basipetospora sp., Candida sp., Chrysosporium sp., Gliocladium sp., Olpitrichum sp., Paecilomyces sp., Staphylotrichum sp., Streptomyces sp., sont des champignons saprophytes communs dans le sol. En revanche, Acremonium sp., Aspergillus niger, Botrytis sp., Brachysporium sp., Candida sp., Fusarium oxysporum, Penicillium sp., Pythium de Baryanum, Rhizopus sp. et Trichoderma sp. peuvent être des parasites.

Fusarium oxysporum a été isolé du sol de la bananeraie sous forme de structure de conservation. C'est un parasite tellurique qui se conserve dans le sol grâce à ses chlamydospores (Mourichon, 2003), considérées comme des structures de conservation extrêmement efficaces lui permettant de résister à des conditions environnementales très défavorables telles que la dessiccation et l'absence d'hôtes (Ploetz et Pegg, 2000).

Les chlamydospores ont la particularité de pouvoir survivre dans le sol en saprophyte sur des débris divers de végétaux (Ploetz et Pegg, 2000). La bananeraie est donc sous sa menace. Il suffit que les conditions lui deviennent favorables pour que sa dissémination et son extension à d'autres zones se fasse très rapidement grâce au réservoir d'irrigation par exemple (Ploetz et Pegg, 2000). Au niveau de la plantation, nous n'avons observé aucun signe extérieur qu'on pourrait attribuer à Fusarium oxysporum responsable de la maladie de Panama. Cependant, la présence de ce pathogène dans la bananeraie peut signifier qu'une analyse fongique préalable du sol n'a pas été faite avant l'installation de la plantation. Le manque de soin et d'hygiène apporté aux outils de travail, le transport dans l'exploitation de terreaux, de plants contaminés par voies mécaniques peuvent être également une des causes de la présence de Fusarium oxysporum. Aspergillus niger a été isolé du sol de la bananeraie. C'est l'une des espèces la plus commune, très répandu dans l'environnement, reconnu comme opportuniste pathogène, sans spécialisation d'hôte (Masayuki et Katsuya, 2010) et selon Howell (2003) toxique et pathogène. Cette espèce est xérophile et exige une activité de l'eau de 0,77 (Fiedler et al., 2001). C'est un champignon mésophile qui se développe à une température optimale qui se situe entre 20 et $40{ }^{\circ} \mathrm{C}$ et peut survivre à $60{ }^{\circ} \mathrm{C}(\mathrm{CRCDG}$, 2007). Cependant, exposé à une température de $63{ }^{\circ} \mathrm{C}$, il ne survit pas (Schuster et al., 2002). Sa présence dans la plantation pourrait être une menace pour les fruits compte tenu $\mathrm{du}$ fait que c'est un champignon responsable de la pourriture des fruits frais après récolte et qui en plus se répand très rapidement dans l'atmosphère avec une grande vitesse de croissance (Samson et al., 2001). 
Tableau 2 : Données analytiques 1 du profil.

\begin{tabular}{|c|c|c|c|c|c|c|c|c|}
\hline \multirow[t]{2}{*}{ Horizons } & \multirow{2}{*}{$\begin{array}{c}\text { Conductivité } \\
(\mu \mathrm{mhos} / \mathrm{cm})\end{array}$} & \multirow{2}{*}{$\begin{array}{c}\text { Carbonate } \\
(\%)\end{array}$} & \multirow{2}{*}{$\begin{array}{c}\mathrm{CO}_{3} \\
(\%)\end{array}$} & \multirow{2}{*}{$\begin{array}{l}\text { Cl } \\
(\%)\end{array}$} & \multirow{2}{*}{$\begin{array}{c}\mathrm{CaCO}_{3} \mathrm{~T} \\
(\%) \\
\end{array}$} & \multirow{2}{*}{$\begin{array}{c}\mathrm{CaCO}_{3} \mathrm{~A} \\
(\%)\end{array}$} & \multicolumn{2}{|c|}{ Humidité (\%) } \\
\hline & & & & & & & Act. & Hygro \\
\hline $\mathrm{A}$ & 0,0001 & 0 & 0,09 & 0,02 & 63,25 & 1,51 & 6,84 & 1,12 \\
\hline B & 0,0001 & 0 & 0,09 & 0,01 & 67,58 & 0,25 & 7,84 & 0,45 \\
\hline$S$ & 0,0001 & 0 & 0,06 & 0,01 & 68,57 & 2,27 & 16,32 & 1,19 \\
\hline $\mathrm{C}$ & 0,0001 & 0 & 0,07 & 0,01 & 65,12 & 1,80 & 12,21 & 2,45 \\
\hline $\mathrm{R}$ & 0,0002 & 0 & 0,09 & 0,01 & 68,45 & 3,55 & 15,04 & 1,36 \\
\hline
\end{tabular}

$\mathrm{Le} \mathrm{pH}$ est homogène tout au long du profil. Il se situe entre 7,7 et 7,9 respectivenent aux horizo $\mathrm{R}$ et $\mathrm{A}$,

Tableau 3: Données analytiques 2 du profil.

\begin{tabular}{|c|c|c|c|c|c|c|c|c|c|c|c|}
\hline \multirow[t]{2}{*}{ Horizons } & \multicolumn{4}{|c|}{ Granulométrie (\%) } & \multirow[t]{2}{*}{ Texture } & \multicolumn{2}{|c|}{$\mathbf{p H}$} & \multirow[t]{2}{*}{$\mathrm{CO}(\%)$} & \multirow[t]{2}{*}{ M.O (\%) } & \multirow[t]{2}{*}{$\mathbf{N}(\%)$} & \multirow[t]{2}{*}{$\mathbf{C} / \mathbf{N}$} \\
\hline & $\mathbf{A}$ & $\mathbf{L F}$ & LG & $\mathbf{S}$ & & Eau & KCl & & & & \\
\hline $\mathrm{A}$ & 3,77 & 26,37 & 13,21 & 46,62 & Limoneuse & 8,6 & 7,9 & 0,26 & 0,45 & 0,35 & 0,76 \\
\hline B & 6,07 & 16,94 & 24,00 & 52,97 & Limono-sableuse & 8,7 & 7,9 & 1,23 & 2,12 & 0,42 & 2,93 \\
\hline S & 9,84 & 27,02 & 22,48 & 40,65 & Limoneuse & 8,5 & 7,8 & 0,31 & 0,54 & 0,14 & 2,25 \\
\hline $\mathrm{C}$ & 6,27 & 20,95 & 07,20 & 65,56 & Limono-sableuse & 8,7 & 7,7 & 1,24 & 2,13 & 0,21 & 5,92 \\
\hline $\mathrm{R}$ & 9,15 & 23,40 & 24,54 & 32,89 & Limoneuse & 8,6 & 7,7 & 1,83 & 3,15 & 0,21 & 8,74 \\
\hline
\end{tabular}


Le genre Trichoderma se retrouve couramment dans le sol comme c'est le cas dans cette bananeraie. Ses propriétés antagonistes sont connues depuis longtemps (Caron, 2002). Trichoderma est efficace lorsqu'on lui permet de s'installer avant l'arrivée des champignons pathogènes, son action est donc préventive (Caron, 2002). Trichodema étant déjà sur le terrain, et Fusarium oxysporum présent sous forme de chlamydospores, Trichoderma pourrait utiliser son mode de vie parasitaire (Caron, 2000) en pénétrant à l'intérieur de l'organe de conservation et/ou en lui injectant des substances (enzymes) qui pourront la détruire. La connaissance de l'humidité du sol est un élément clé pour suivre et comprendre la croissance de la végétation, pouvoir améliorer la prévision des caractéristiques de ruissellement (modélisation) dont dépendent les risques d'inondation ainsi que la gestion des ressources en eau. L'humidité du sol est aussi un paramètre d'alerte pour la désertification (Shortt et al., 2011)

La conductivité électrique définie la quantité totale en sels solubles correspondant à la salinité globale du sol, elle dépend de la teneur et de la nature des sels solubles présents dans ce sol (Guessoum, 2001). Les très faibles valeurs de conductibilité enregistrées, indiquent une absence de source géologique de sels dans la zone d'étude. Le sol est donc très faiblement salé et sans incidence sur la culture. Ce résultat est confirmé par l'absence de carbonate et par les très faibles taux de bicarbonates et de chlorure. Par contre, le test à l'acide chlorhydrique de nos différents échantillons, révèle une très forte effervescence, ce qui indique que le sol de la bananeraie est extrêmement calcaire. Ce résultat est confirmé par le dosage du calcaire total dont les taux sont > 50\% (LANO, 2000). Son absence aurait eu pour conséquence une acidification progressive du sol. Le calcaire, dans une certaine proportion, est donc un élément important qui participe à la fertilité du sol. Selon Bourguignon (2002), lorsque le taux de calcaire actif est inférieur à $4 \%$, il n'a pas ou peu d'action chlorosante. C'est à partir de $7 \%$, qu'il apparaît légèrement chlorosant, ce qui n'est pas le cas du sol de la bananeraie. C'est une caractéristique stable du sol. La présence de calcaire confère au sol des caractéristiques spécifiques en termes de comportement physique et chimique et influe sur son activité biologique (Baize, 2000). L'acidité actuelle est la plus importante car c'est elle qui a un effet sur la végétation et la microflore. Le $\mathrm{pH}$ (eau) est voisin de la neutralité, donc favorable à la culture du bananier, ce qui va permettre l'assimilation de la plupart des éléments nutritifs (Soltner, 2000). Le $\mathrm{pH}_{(\mathrm{KCl})}$ est un $\mathrm{pH}$ «théorique » qui permet de connaître l'acidité potentielle du sol. Il est toujours inférieur au $\mathrm{pH}_{\text {(eau). L'écart }}$ entre les deux varie de 0,5 à 1,5 (Baize, 2000), ce qui se vérifie au cours de nôtre étude. La matière organique intervient sur les propriétés physiques du sol et réagit en collaboration avec l'azote dans la nutrition des végétaux. Le bananier exige beaucoup d'azote. La dose préconisée d'apport d'azote est de 450 - $600 \mathrm{~kg} / \mathrm{ha} /$ cycle (Skiredj et al, 2012).

Selon Calvet (2003), les teneurs en azote qui sont comprises entre $0,15 \%-0,25 \%$ et $>0,25 \%$ signifient que le sol est respectivement riche en azote en profondeur et très riche en surface. La teneur en azote total des sols de culture varie de 0,1 à $0,5 \%$, celle de la bananeraie se situe entre 0,14 et $0,42 \%$.

La matière organique est le constituant du sol le plus important, presque la moitié de la matière organique du sol se présente sous une forme stable appelée humus, l'autre moitié est constituée de matière décomposée 
telle que les résidus de cultures, les organismes morts constitués de la flore et de la faune du sol (Girard et al., 2005).

Elle a également des effets favorables sur les propriétés physiques du sol en améliorant sa perméabilité, sa stabilité, et sa capacité de rétention en eau (Soltner, 2000).

Le sol de la bananeraie est moyennement pourvu en matière organique au niveau des horizons $\mathrm{B}, \mathrm{C}$ et $\mathrm{R}$ car les taux sont compris entre 2 et $3 \%$ de matière organique et très pauvre en matière organique au niveau de $A$ et $\mathrm{S}$ parce que le pourcentage de matière organique est $<1,4 \%$ (LANO, 2000).

Selon Girad et al. (2005), le rapport $\mathrm{C} / \mathrm{N}$ est un indicateur de l'activité biologique des sols. Il renseigne sur le degré d'évolution de la matière organique, l'activité biologique et le potentiel de fourniture d'azote par le sol (minéralisation).

Selon LCA (2008), le rapport $\mathrm{C} / \mathrm{N}<10$ traduit une bonne décomposition de la matière organique qui conduit à la minéralisation de la matière organique, ce qui est en accord avec les résultats obtenus à l'issu de l'étude des horizons du profil de la bananeraie.

\section{Conclusion}

Cette étude a permis d'identifier plusieurs champignons au sein de la bananeraie. La présence de Fusarium oxysporum est inquiétante bien qu'elle soit sous sa forme de conservation. Ce champignon sous sa forme active est responsable de la maladie de Panama dont l'impact concerne les parties souterraines et ariennes de la plantes, à savoir les racines, les cornes et les gaines foliaires. En général, les bananiers atteints par la fusariose meurent après flétrissement. La bananeraie est donc sous une grande menace. L'étude pédologique quant à elle, a révélée les propriétés physiques et chimiques qui caractérisent un sol pour la culture de la banane. Ce travail a permis de mettre en évidence l'importance d'une analyse fongique préalable avant l'installation d'une plantation industrielle.

\section{REFERENCES}

Baize D. 2000. Guide des Analyses en Pédologie (2e édn, Revue et Augmenté). Editions INRA ; 99p.

Barnett H, Hunter Barry B. 1972. Illustrated Genera of Imperfecti Fungi (3e édn). Burgess Publishing Company: Minneapolis, USA, 241p.

Baudouin JP, Demol J, Louant BP, Marechal R, Mergeai G, Otoul E. 2002 L'Amélioration des Plantes. Application aux Principales Espèces Cultivées en Régions Tropicales. Les Presses Agronomiques de Gembloux : Gembloux ; 252.

Black CA, Evan DD. 1965. Methods of soil analysis, American Society of Agronomy. Agronomy, 9: 917-918.

Bonfils P. 1967. Méthodes d'analyses des sols. Service d'étude des sols, $\mathrm{n}^{\circ} 91$, $156 \mathrm{p}$.

Bourguignon C. 2002. Le sol, la Terre et les Champs. Ed. Sang de la Terre : Paris.

Cabrera Cabrera J, Cubas AP, Jaizme C, Vega J, Cepero L. 2010. Lutte intégrée et production organique en culture bananière : étude de cas des Iles Canaries, Étude de Cas sur la Banane. Guide Numéro 5, 8p.

Calvet R. 2003. Le Sol: Propriétés et Fonctions ( $1^{\mathrm{e}}$ édn). France Agricole, Dunod : Paris ; 456.

Caron J. 2002. Le pouvoir antagoniste de Trichoderma. Conférence présentée lors des journées horticoles régionales à StRémi, 4p.

Champion R. 1997. Identifier les champignons transmis par les semences. INRA (Institut National de la Recherche 
Agronomique) 147, rue de l'Université, 75338 Cedex 07, 398p.

Cirad. 2002. Mémento de l'agronome: Agriculture, 1646p.

Cirad. 2010. Bananes-8p-A4-v18.indd.

CNRA (Centre National de Recherche Agronomique). 2015. Programme

Plantain, Bananes, Ananas, $3 p$.

CRCDG (Centre de Recherche sur la Conservation des Documents Graphiques). 2007. Moisissures et biens culturels. Ministère de la culture et de la communication, France.

Drouineau G. 1940. Dosage rapide du calcaire actif des sols. Nouvelles données sur la répartition et la nature des fractions calcaires. Annales Agron., 12(44): 1-450.

Duchaufour P. 1970. Précis de Pédologie. Masson, Paris ; 189.

FAO. 2003. Projections à Moyen Terme pour les Produits Agricoles de Base. CCP: Rome.

Fiedler K, Schutz E, Geh S. 2001. Detection of microbial volatile organic compounds (MVOCs) produced by moulds on variuos materials. International Journal of Hygiene and Environmental Health, 204: 111-121.

Gilles C. 2012. La culture de la banane 8p.

Girard MC, Walter C, Remy JC, Berthelin J, Morel JL. 2005. Sols et Environnement, (2e édn), Editions Dunod : Paris ; 28.

Guessoum A. 2000. L'effet de l'irrigation sur la salinité du sol dans la région de Saada Biskra. Thèse ing, Agro, Univ Batna, $50 \mathrm{p}$.

Howell CR. 2003. Mechanisms employed by Trichoderma species in the biological control of plant diseases: the history and evolution of current concepts. Plant Diseases: Journals of American Phytopathological Society 87: 4-10.
Kjeldah J. 1883. Méthode pour déterminer l'azote organique. Revue Allemande de Chimie Analytique.

LCA (Laboratoire de Conseils et d'Analyses). 2008 : Guide pratique : comprendre et utiliser les analyses agroenvironnementales. Laboratoire de Conseils et d'Analyses ; 30p.

LANO (Laboratoire Agronomique de Normandie). 2000. Analyse de Terre. Chambre d'Agriculture du Calvados, 37p.

Lapeyre de Bellaire L, Abadie C, Carlier J, Ngando J, Gert HJ. 2010. ENDURE Étude de Cas sur la Banane: Les cercosporioses des bananiers (Mycosphaerella spp) : vers une lutte intégrée. Guide Numéro 2 (French), 6p.

Lassoudière A. 2007. Le Bananier et sa Culture. Editions Quæ: Versailles, France.

Maignien R. 1980. Manuel pour la Description des Sols sur le Terrain: ORSTOM ; Paris ; 2-112.

Masayuki M, Katsuya G. 2010. Aspergillus: molecular biology and genomics. Ed Caister Academic Press : Norfolk (England); 238.

Mourichon X. 2003. Analyse du Risque Phytosanitaire. Cirad Référence : BAN $\mathrm{C}_{4} ; 26$.

PACIR (Programme d'Appui au Commerce et à l'Intégration Régionale). 2013. Evaluation du potentiel à l'exportation des fruits tropicaux. 159p.

Ploez RC, Pegg G. 2000. Fungal Disease of the root, Corm and Pseudostem: Fusarium wilt. In Disease of Banana. Edition. D. R. Jones : Abaca and Ensete ; 143-159.

Pochon J, Tardieux P. 1962. Techniques d'Analyse en Microbiologie du Sol. Ed. de la Tourelle : Saint-Mandé ; $108 \mathrm{p}$.

Samson RA, Houbraken J, Summerbell RC, Flannigan B, Miller JD. 2001. Common 
and important species of fungi and actinomycetes in indoor environments. In Microorganisms in Home and Indoor Work Environments. Taylor \& Francis: New York; 287-292.

Schuster E, Dunn-Coleman N, Frisvad JC, Van Dijck PW. 2002. On the safety of Aspergillus niger. a review. Appl. Microbiol. Biotechnology, 59(465): 426435.

Shortt R, Verhallen A, Pam Fisher P. 2011. Surveiller l'humidité du sol pour améliorer les décisions d'irrigation. Fiche technique : 753 /560 25p.
Skiredj A, Walali DML, El Attir H. 2012. La culture du bananier sous serre au Maroc. www.legume-fruit-maroc.com 6p.

Soltner D. 2000. Les Bases de la Production Végétale, T1: le Sol et son Amélioration $\left(22^{\mathrm{e}}\right.$ édn). Editions Sciences et Techniques Agricoles "Le clos Lorelle" : 49130 Saint-Gemmes-Sur-Loire, 472p.

Swennen R, Vuylsteke D. 2001. Bananier In RAEMARKERS HR : Edition Agriculture en Afrique Tropicale DGCI, Bruxelles, 611Z636. 